\title{
Quantum Brownian motion on a lattice
}

\author{
Ping Sheng and Zhao-Qing Zhang* \\ Exxon Research \& Engineering Company, Route 22 East, Annandale, New Jersey 08801
}

(Received 23 March 1993)

\begin{abstract}
For a quantum particle on a one-dimensional lattice, the dynamics under the influence of a timedependent, white-noise potential is shown to differ significantly from the classical diffusive behavior. In particular, the square of the distance displaced from origin is proportional to $t^{0.5-0.6}$. The origin of this dispersive behavior is traced to the special properties of the force-force correlation function, which integrates to zero and has a magnitude varying as $t^{-0.5}$. These characteristics are attributed to the continuous spread of the quantum wave packet as well as its interaction with the discrete lattice.
\end{abstract}

Quantum Brownian motion (QBM) may be defined as the dynamics of a quantum particle under the influence of a time-dependent, white-noise potential. As one of the simplest generic stochastic problems, QBM is the quantum counterpart to the classical Brownian motion that gives rise to diffusive transport, and it is directly relevant to the transport of quantum excitations in an environment involving localized traps. In a slightly modified form, QBM is also relevant to the directed polymer problem and the problem of quantum tunneling probability of a strongly localized electron. ${ }^{1-3}$ In a lattice QBM problem, the scaling behavior of the width of the wave function $\left\langle x^{2}(t)\right\rangle$, a second-moment quantity, is known to follow a diffusive behavior from both analytic ${ }^{3-5}$ and numerical works ${ }^{2,6}$ due to the unilarity condition of the Schrödinger's equation. A superdiffusive behavior was found for a continuous model. ${ }^{7}$ However, the scaling behavior of the square of the distance displaced from the origin, $\langle x(t)\rangle^{2}$, which involves the fourth moment of the wave function, has been the focus of many recent studies $^{1-3,6}$ because no analytic solution is known. In one dimension, numerical results suggest that $\langle x(t)\rangle^{2}$ follows a subdiffusive behavior ${ }^{2,6}$ and scales as $t^{0.5-0.6}$. In this work, QBM is studied through the numerical solution of stochastic Schrödinger's equation on a one-dimensional lattice using a new numerical scheme. We have also found that $\langle x(t)\rangle^{2}$ scales as $t^{0.5-0.6}$. The motivation of this work is to understand the origin of this power law. Through the analysis of the momentum and force correlations, the origin of this dispersive transport behavior is traced to the combined effect of wave scattering from the lattice and the inherent spreading of the wave packet that represents the quantum particle.

The starting point of our consideration is the Schrödinger's equation

$$
i \frac{\partial \varphi}{\partial t}=H \varphi
$$

where we have set $\hbar=1$, mass of the particle $m=\frac{1}{2}$, so that $\hbar^{2} / 2 m=1$. For a one-dimensional lattice with lattice constant $=1$ the Hamiltonian in the tight-binding approximation can be written as

$$
H=-\Delta^{(2)}+W \eta(i, t) \text {. }
$$

In Eq. (2), $\Delta^{(2)}$ denotes the discrete Laplacian $\Delta^{(2)} \varphi(i)=\varphi(i+1)-2 \varphi(i)+\varphi(i-1), i$ is the lattice-site index, $W$ is the magnitude of the random potential, and $\eta$ is a random function with values in the interval $[-1,1], \overline{\eta(i, t)}=0$, and

$$
\overline{\eta(i, t) \eta\left(j, t^{\prime}\right)}=\delta_{i j} \delta\left(t-t^{\prime}\right),
$$

where the overbar denotes ensemble averaging. At time $t=0$, the quantum particle is represented by a Gaussian packet centered at $i=0$ with a width $\sigma=10$ :

$$
\varphi(i, t=0)=\frac{1}{\sqrt{2 \pi} \sigma} \exp \left(-i^{2} / 2 \sigma^{2}\right) .
$$

The subsequent space-time evolution of the packet is calculated numerically by a stable implicit scheme which also preserves the unitary of the wave function. By using $n$ to denote the time step index, the scheme is given by

$$
\begin{aligned}
\varphi(i, n+1)-\varphi(i, n)=\frac{i \Delta t}{2}\{[ & \left.\Delta^{(2)} \varphi(i, n+1)+\Delta^{(2)} \varphi(i, n)\right] \\
- & \frac{W}{2}[\eta(i, n)+\eta(i, n+1)] \\
& \times[\varphi(i, n)+\varphi(i, n+1)]\} .
\end{aligned}
$$

Equation (5) is easily recognized as an extension of the Crank-Nicolson method ${ }^{8}$ where the particular discretization scheme of the $\eta(i, t) \varphi(i, t)$ term is necessitated by the consideration of unitary conservation.

Two types of expectation values can be obtained from the long-time solutions of Eqs. (1) and (2). The first type is the quantum expectation values. These are defined by

$$
\begin{aligned}
& \langle x(t)\rangle=\sum_{i} i|\varphi(i, t)|^{2},\langle p(t)\rangle=-i\langle\varphi|[x, H]| \varphi\rangle / 2, \\
& \langle f(t)\rangle=-W\langle\varphi|\eta(i+1, t)-\eta(i-1, t)| \varphi\rangle / 2,
\end{aligned}
$$

and

$$
\langle E(t)\rangle=\langle\varphi|H| \varphi\rangle .
$$

Here $p$ denotes the momentum operator, $f$ the force, $E$ the energy, and $[x, H]$ the commutator of position operator $x$ and $H$. The quantum expectation values $\langle x(t)\rangle$, 
$\langle p(t)\rangle$, and $\langle f(t)\rangle$, should satisfy the classical equations of motion: ${ }^{9}$

$$
\begin{aligned}
& \frac{1}{2} \frac{d\langle x(t)\rangle}{d t}=\langle p(t)\rangle, \\
& \frac{d\langle p(t)\rangle}{d t}=\langle f(t)\rangle,
\end{aligned}
$$

where the factor $\frac{1}{2}$ in Eq. (6a) is from the mass of the particle. By using $\Delta t=0.15$ [in units of $2 m \times$ (lattice con$\left.\operatorname{stant})^{2} / \hbar\right]$, we have verified the conservation of unitarity, as well as Eqs. (6a) and (6b), to a high degree of accuracy. From the quantum expectation values, which give the ex-

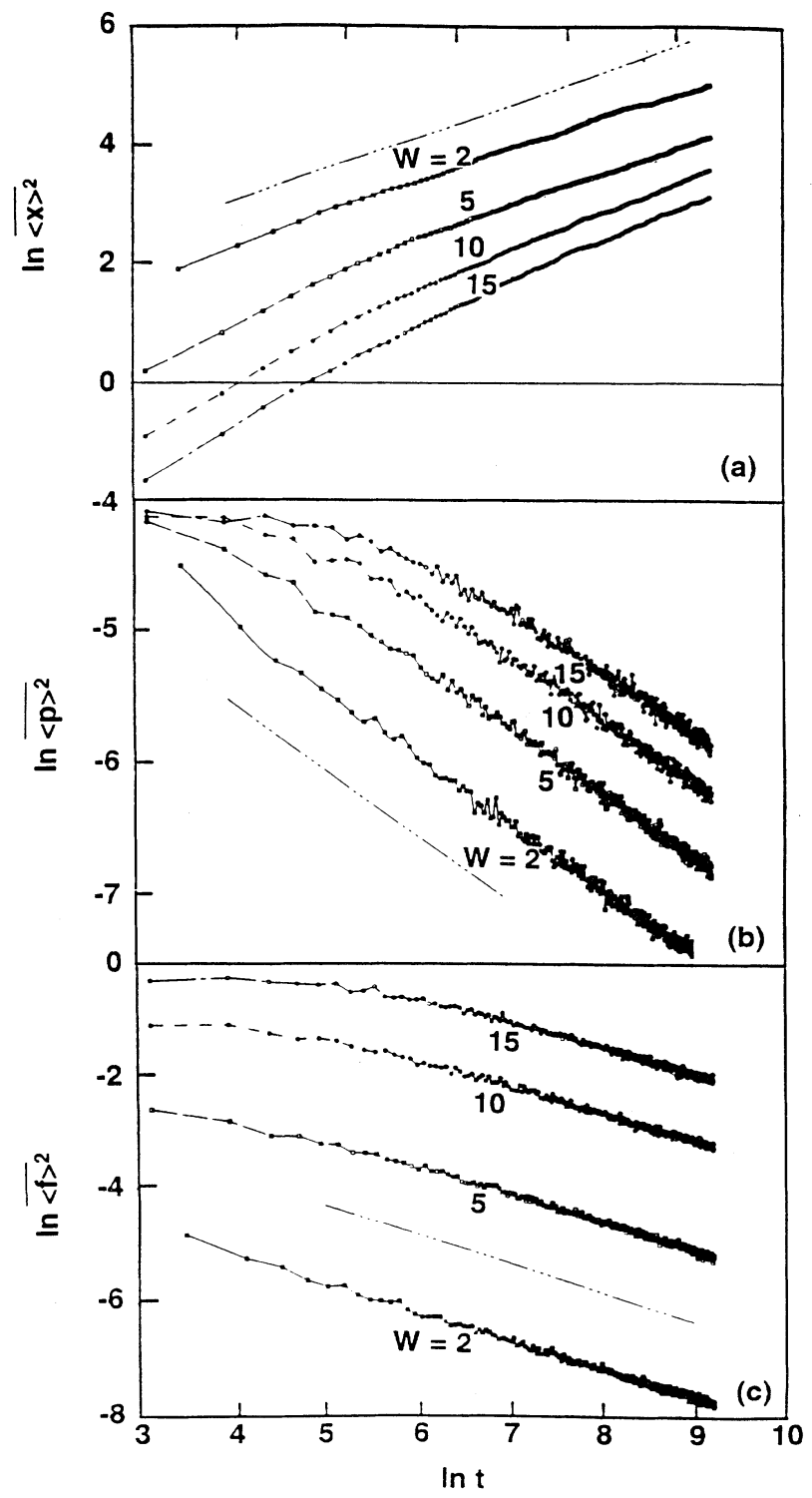

FIG. 1. Time dependence of (a) $\overline{\langle x(t)\rangle^{2}}$, (b) $\overline{\langle p(t)\rangle^{2}}$, and (c) $\overline{\langle f(t)\rangle^{2}}$ for $W=2,5,10$, and 15. The dot-dashed line in (a) represents a power-law of $t$ with the exponent value of 0.55 . The dot-dashed lines in (b) and (c) represent a power of $t$ with the exponent value of -0.5 . Data points represent the averages of 1200-1800 configurations. pected values for the position, momentum, and force measurements on a quantum particle, one can obtain the analogous classical expectation values of $\overline{\langle x(t)\rangle^{2}}$, $\overline{\langle p(t)\rangle^{2}}$, and $\overline{\langle f(t)\rangle^{2}}$. Ensemble averages of these quantities from 1200 to 1800 configurations are shown in Fig. 1 for $W=2,5,10$, and 15 . It is seen that in the large $t$ limit, $\overline{\langle x(t)\rangle^{2}} \propto t^{\alpha}$, where $\alpha=0.5-0.6$, and $\overline{\langle p(t)\rangle^{2}}$ and $\overline{\langle f(t)\rangle^{2}}$ both exhibit $t^{-1 / 2}$ behavior. Our result on $\langle x(t)\rangle^{2}$ is noted to agree with prior estimates. ${ }^{2,6}$ However, both the cause of this behavior and the $\overline{\langle p(t)\rangle^{2}}$ and $\overline{\langle f(t)\rangle^{2}}$ characteristics are yet to be understood. Below we examine these issues in more detail.

Since the quantum expectation values of $x, p$, and $f$ satisfy the classical equations $(6 a)$ and $(6 b)$, the behaviors of $\langle p(t)\rangle$ and $\langle f(t)\rangle$ are thus crucial in understanding the distinct properties of QBM from those of the classical case. If $\langle p(t)\rangle=\eta(t)=$ white noise, then we recover the classical behavior ${ }^{3} \overline{\langle x(t)\rangle^{2}} \propto t$. If, on the other hand, $\langle f(t)\rangle=\eta(t)=$ white noise, then $\overline{\langle x(t)\rangle^{2}}$ should vary as $t^{3}$ as in the case of a classical random walker with no dissipation $^{10}$ (so the energy of the particle can increase indefinitely). Both cases can be easily verified by the solutions of Eqs. (6a) and (6b). In the present case, however, the decrease of $\overline{\langle f(t)\rangle^{2}}$ as $t^{-1 / 2}$ can be understood physically as resulting from wave-packet spreading. That is, if we evaluate the variance $l^{2}=\overline{\left\langle(x-\langle x\rangle)^{2}(t)\right\rangle}=\overline{\left\langle x^{2}\right\rangle}$ $-\overline{\langle x\rangle^{2}}$ of the wave packet, it is found to be proportioned to $t$ as shown in Fig. 2. It should be noted here that $\overline{\left\langle x^{2}\right\rangle}$ is a purely quantum quantity that has no classical analog. Our results on the long-time behavior of $\overline{\left\langle x^{2}\right\rangle} \sim t$ agree accurately with prior analytical solutions of the problem. ${ }^{4,5}$ Since the effective force $\langle f(t)\rangle$ is the result of averaging the derivative of the random potential over the width $l$ of the wave packet, it follows that as $t$ increases, $\langle f(t)\rangle$ should decrease as $1 / \sqrt{l}$. Since $l^{2} \propto t, \overline{\langle f(t)\rangle^{2}}$ therefore varies as $1 / \sqrt{t}$.

The above argument, however, sheds no light on the

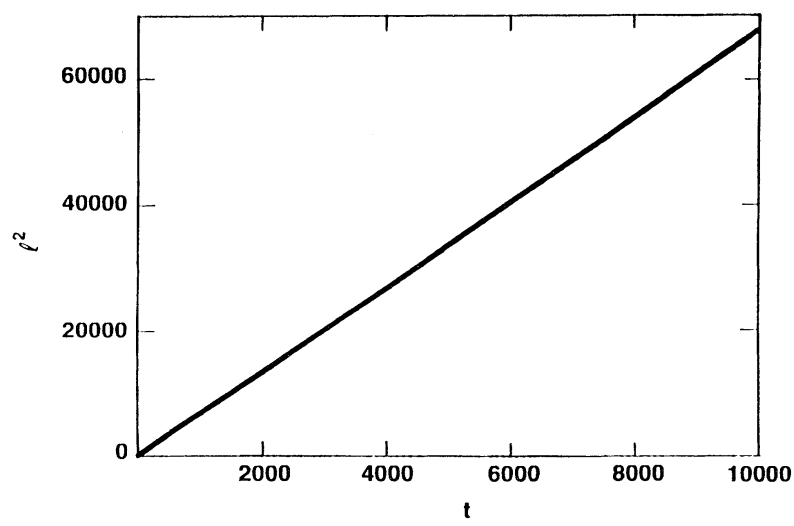

FIG. 2. Time variation of the variance $l^{2}$ of the wave function as defined in the text. An accurate linear dependence on $t$ is seen. Data represent the average over 1000 configurations. The value of $W$ used is 2 . 


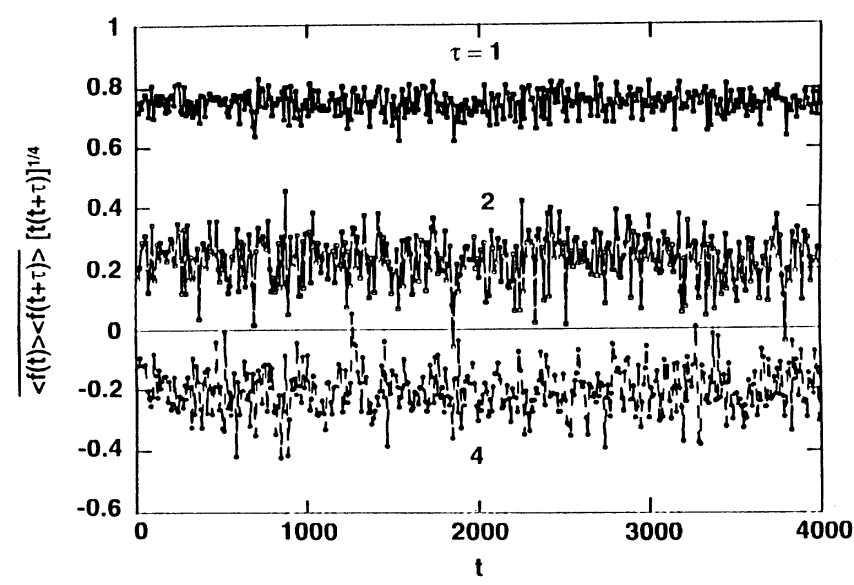

FIG. 3. Plot of the force-force correlation function multiplied by $[t(t+\tau)]^{1 / 4}$, averaged over 150 configurations. The constant trend is unmistakable.

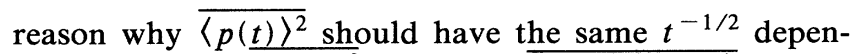
dence as $\frac{\langle f(t)\rangle^{2}}{\left\langle\text { If } \overline{\langle f(t)\rangle\left\langle f\left(t^{\prime}\right)\right\rangle}\right.}=$ const $\times \delta\left(t^{\prime}-t\right) / \sqrt{t}$, then $\overline{\langle p(t)\rangle^{2}}$ would vary as $t^{1 / 2}$, and the behavior of $\overline{\langle x(t)\rangle^{2}}$ is at least superdiffusive, i.e., $t^{\alpha}$ with $\alpha>1$. The present $\overline{\langle p(t)\rangle^{2}} \infty t^{-1 / 2}$ behavior thus implies a nontrivial force-force correlation function that differs from $\delta\left(t^{\prime}-t\right)$.

To calculate the force-force correlation function, we first verify that it can be written in the form

$$
\overline{\langle f(t)\rangle\left\langle f\left(t^{\prime}\right)\right\rangle}=H_{0} h\left(t^{\prime}-t\right) /\left(t t^{\prime}\right)^{1 / 4},
$$

where $H_{0}=\overline{\langle f(t)\rangle^{2}} \sqrt{t}$ is a constant evaluated at the $t \rightarrow \infty$ limit where $\overline{\langle f(t)\rangle^{2}} \propto 1 / \sqrt{t}$. The fact that $\left(t t^{\prime}\right)^{1 / 4} \overline{\langle f(t)\rangle\left\langle f\left(t^{\prime}\right)\right\rangle}$ indeed behaves as a constant for a fixed value of $\tau=t^{\prime}-t$ is shown in Fig. 3 for $W=7, \tau=1$, 2 , and 4. One hundred fifty configurations were used. While there are fluctuations, the flat trend is unmistakable. Similarly, the momentum-momentum correlation function may be expressed as

$$
\overline{\langle p(t)\rangle\left\langle p\left(t^{\prime}\right)\right\rangle}=G_{0} g\left(t^{\prime}-t\right) /\left(t t^{\prime}\right)^{1 / 4},
$$

with $G_{0}=\overline{\langle p(t)\rangle^{2}} \sqrt{t}$, defined similarly as $H_{0}$ and which

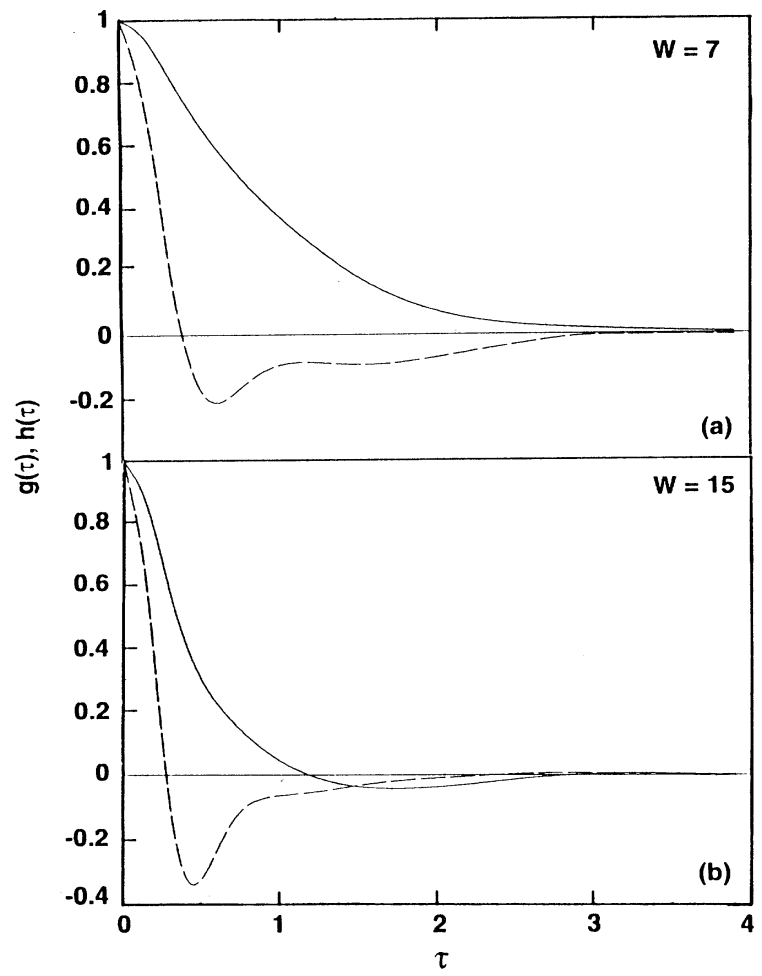

FIG. 4. The momentum-momentum correlation function (solid lines) and the force-force correlation function (dashed lines) plotted as a function of $\tau$ for two values of W. (a) $W=7$, (b) $W=15$.

may also be verified to be a constant. Figure 4 gives the behaviors of $h(\tau)$ and $g(\tau)$ for $W=7$ and 15, obtained by both configurational averaging and averaging over $t$. The solid lines in Fig. 4 depict $g(\tau)$, and the dashed lines $h(\tau)$. It is seen that whereas $g(\tau)$ decays nearly exponentially, $h(\tau)$ has a very pronounced negative lobe. What is special about $h(\tau)$, however, is that it has compact support, i.e., $h(\tau)=0$ for $\tau>\tau_{0}(W)$. Moreover, the integral of $h(\tau), 0 \leq \tau \leq t_{0}$, is accurately zero (to three decimal places) for all values of $W$ evaluated. This special property means that

$$
\begin{aligned}
\overline{\langle p(t)\rangle^{2}} & =\int_{0}^{t} \int_{0}^{t} \overline{\left\langle f\left(t_{1}\right)\right\rangle\left\langle f\left(t_{2}\right)\right\rangle} d t_{1} d t_{2} \\
& \cong 2 H_{0} \int_{0}^{t} d \tau h(\tau) \int_{\tau}^{t} T^{-1 / 2} d T+\frac{1}{2} H_{0} \int_{0}^{t} d \tau \tau h(\tau) \int_{\tau}^{t} T^{-3 / 2} d T+\cdots \\
& =4 H_{0} \sqrt{t} \int_{0}^{t} d \tau h(\tau)-4 H_{0} \int_{0}^{t} d \tau \sqrt{\tau} h(\tau)+\frac{1}{2} H_{0} \int_{0}^{t} d \tau \tau h(\tau) \int_{\tau}^{t} T^{-3 / 2} d T+\cdots
\end{aligned}
$$

Since $t \gg \tau_{0}$, the first term is zero, and from the second and third terms one obtains

$$
\overline{\langle p(t)\rangle^{2}}=\frac{-H_{0}}{\sqrt{t}} \int_{0}^{\tau_{0}} d \tau \tau h(\tau)-3 H_{0} \int_{0}^{\tau_{0}} d \tau \sqrt{\tau} h(\tau)+\cdots,
$$

i.e., the leading nonconstant term of $\overline{\langle p(t)\rangle^{2}}$ is propor- tional to $1 / \sqrt{t}$, in agreement with observed results. A similar analysis yields the result that $\overline{\langle x(t)\rangle^{2}} \propto t^{0.5-0.6}$ because the integral of $g(\tau)$ from 0 to $t$ gives $t^{0-0.1}$.

Figure 5 gives the variation of $\tau_{0}^{-1}(W)$ summarized from the calculations of $h(\tau)$ for various values of $W . \tau_{0}$ represents the underlying memory time of the QBM. The quadratic behavior of $\tau_{0}$ at small $W$ is expected on the 


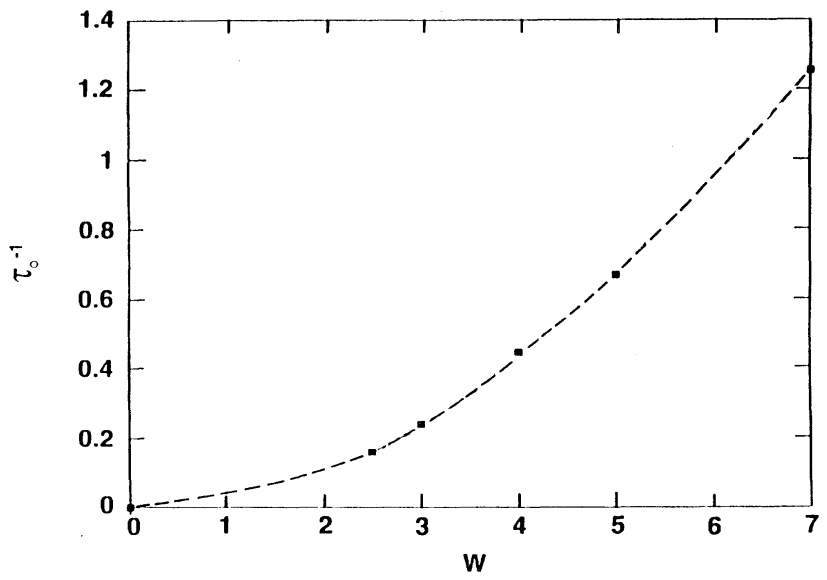

FIG. 5. Plot of $\tau_{0}^{-1}$ as a function of $W . \tau_{0}$ represents the correlation time for the quantum Brownian motion. Dashed line is to guide the eye.

basis of symmetry of the problem under the transformation of $W \rightarrow-W$.

The physical reason for the special characteristics of $h(\tau)$ lies in the interaction of the quantum wave with the discrete lattice, which gives rise to an energy band. Consequently, the quantum particle can have no net energy increase as soon as the states in the band are equally occupied. A quick check of $\overline{\langle E(t)\rangle}$ shows this to be indeed the case: while there are fluctuations, the overall average stays constant at the center of the band. Moreover a well-known effect of a discrete lattice is the creation of positive and negative curvatures in the dispersion relation of an electron at the lower and upper band edges, respectively. These curvatures correspond exactly to positive and negative "effective mass" 11 at the band edges. In this context the negative correlation lobe in $h(\tau)$ may be understood as the result of effective-mass sign change as the energy of the particle moves between the upper and the lower band edges (thus changing the sign of the force as well), in analogy with the well-known Bloch oscillation ${ }^{11}$ effect of an electron on a lattice. The existence of such significant lattice effects means that in contrast to the classical Brownian motion, the continuum version of the QBM could be drastically different from the lattice version. However, the lattice QBM should be physically observable through experiments involving the trapping of quantum excitations in an atomic lattice.

From the above discussion it is easily concluded that the passage of quantum to classical random walk requires the removal of both the wave effect and the dispersion effect. In the context of the present model this might be achieved by the addition of a static random potential whose magnitude is much larger than $W$. In that case the wave packet will be localized in the Anderson sense by the static disorder, and the effect of the dynamic random potential is then to cause random hoppings of the localized packet. Further investigations along this direction are presently under way.

This work is supported by ONR Contract No. N00014-88-K-0003.
*Also at Institute of Physics, Academia Sinica, Beijing, China.

${ }^{1}$ S. Feng, L. Golubovic, and Y. C. Zhang, Phys. Rev. Lett. 65, 1028 (1990).

${ }^{2}$ E. Medina, M. Kardar, and H. Spohn, Phys. Rev. Lett. 66, 2176 (1991).

${ }^{3}$ L. Sand and M. Kardar, Phys. Rev. A 45, 8859 (1992).

${ }^{4}$ A. A. Ovchinnikov and N. S. Erikhman, Zh. Eksp. Teor. Fiz. 67, 1474 (1975) [Sov. Phys. JETP 40, 733 (1975)].

${ }^{5}$ A. Madhukar and W. Post, Phys. Rev. Lett. 39, 1424 (1977).

${ }^{6}$ J. P. Bouchard, D. Touati, and D. Sornette, Phys. Rev. Lett. 68, 1787 (1992).
${ }^{7}$ L. Golubovac, S. Feng, and F. Zeng, Phys. Rev. Lett. 16, 2115 (1991).

${ }^{8}$ G. D. Smith, Numerical Solution of Partial Differential Equation (Clarendon, Oxford, 1985).

${ }^{9}$ L. I. Schiff, Quantum Mechanics, 3rd ed. (McGraw-Hill, New York, 1968), p. 174.

${ }^{10}$ G. E. Uhlenbeck and L. S. Ornstein, Phys. Rev. 36, 823 (1930); A. M. Jayannavar and N. Kumar, Phys. Rev. Lett. 48, 553 (1982).

${ }^{11}$ N. W. Aschcroft and N. D. Merimen, Solid State Physics (Saunders College, Philadelphia, 1976), p. 224. 